\title{
Comments regarding Hiramoto et al.
}

\author{
Leonardo Vinicius Monteiro de Assis ${ }^{1}\left[\right.$. Ana Maria de Lauro Castrucci ${ }^{2}$
}

Received: 7 June 2021 / Accepted: 26 August 2021 / Published online: 1 September 2021

(c) The Author(s), under exclusive licence to European Photochemistry Association, European Society for Photobiology 2021

We have read the paper by Hiramoto et al. [1] published in this Journal. In the next lines, we provide some arguments that mainly disagree with the authors' points and conclusions.

The experimental design used by the authors raises several questions:

1) Since the eyes were exposed, two important issues should have been addressed: (a) Were artificial drops or physiological saline added to the eyes to avoid eye damage? (b) The irradiance and exposure duration are currently missing, and such information is important for reproducibility.

2) The authors mention the following sentence about the experimental protocol: "This procedure disrupted the circadian rhythm of the clock gene Per2, which exhibits increased expression by approximately 10-12 times (data not shown)". The authors should have provided experimental evidence of circadian disruption and not only given a statement. Moreover, this statement seems to contradict the results shown in Fig. 2.

3) In addition, the study by Hiramoto et al. [1] shows the following experimental limitations:

a. The experimental protocol that consists of approximately 156 rounds of UV and anesthesia results in an unrealistic condition mainly based on high UV dose and frequent exposure to anesthesia. Such experimental paradigm created a non-physiological condition. To circumvent this issue, non-exposed group, sham mice, should have been used. This

Leonardo Vinicius Monteiro de Assis

leonardo.deassis@uni-luebeck.de

$\triangle$ Ana Maria de Lauro Castrucci

amdlcast@ib.usp.br

1 Center for Brain, Behavior, and Metabolism, Institute of Neurobiology, University of Lübeck, Lübeck, Germany

2 Department of Physiology, Institute of Biosciences, University of São Paulo, São Paulo, Brazil would help assess the impact of the experiment design into the animal's physiology.

b. The authors consider visible light as innocuous. This concept is no longer sustained as several studies have clearly shown the deleterious effects of visible light [2-4]. The non-irradiated group is crucial in this sense. Unfortunately, nor the irradiance neither the dosage of visible light are provided.

c. The authors state that no damage to the eye was found and they referenced another paper, with a very similar strategy, by the same group; however, no information about eye damage assessment is found in the cited paper [5]. In this study, only histological analyses of the skin were done, but no data about eye histology are provided.

d. Another critical issue is the year-long drug intracerebral delivery. Such strategy can be very difficult to handle due to its long duration. Information about animal health and if this procedure affected other parameters in mice is not provided. This year-long drug delivery, as mentioned before, suffers from the same lack of justification and physiological relevance.

In addition to the limitations highlighted above, the authors make statements that cannot be sustained by their data. We highlight and comment them next.

"This study shows that long-term UVA eye irradiation disrupts the circadian rhythm of clock genes and decreases the memory and learning ability in mice". This statement cannot be sustained with the current data provided as no temporal circadian evaluation was done in addition to a highly disruptive experimental protocol used without appropriate controls.

"It is important to protect the eyes from UVA exposure, especially in human, it is important to protect the eyes from UVA exposure, since UVA effects by brain not eye itself. Any chronic stress that is physical in nature will likely have outcomes similar to those of eye exposure to UVA. However, the damage caused by long-term exposure to UVA 
accumulates over time in humans, increasing the likelihood of decrease in memory and learning ability". This statement cannot be sustained with the current set of data presented. Second, the experimental protocol and UVA dosage used are completely artificial with no parallel to humans, and therefore, its translational aspect is virtually impossible.

"Moreover, the small ophthalmic dimensions in mice, compared to those in humans, facilitate deeper UVA penetration. On the contrary, humans are diurnal and exhibit lower sensitivity to UVA than mice". Such statement is not referenced and a proper citation must be provided. Moreover, the second sentence is not entirely accurate as humans are more sensitive to light-induced effects as S-phase of skin cells take place in late afternoon while in mice this phase happens at night (reviewed in [6]).

"Therefore, compared with mice, the depression in learning ability caused by long-term UVA eye irradiation is not noticeable in humans to UVA than mice". The authors make an important statement, but do not provide any evidence for this.

"Circadian rhythm is modulated by the transaction of two or more clock genes having transcriptional activities". In addition to a non-idiomatic sentence, a common problem across the manuscript, circadian clock modulation is far more complex than only transcriptional activity. There exists circadian modulation in several steps from DNA being transcribed into RNA and then being translated into protein, which may still undergo post-translational modifications (reviewed in [7]).

Taken altogether, we are not commenting on the quality of the data presented, but rather we disagree with the experimental model, lack of proper controls, and consequently, the conclusions drawn by the authors.

Funding Castrucci's lab is supported by the Sao Paulo Research Foundation (FAPESP, 2017/24615-5 and 2018/14728-0) and by the National Council of Technological and Scientific Development (CNPq 303070/2015-3).

\section{Declarations}

Conflict of interest The authors declare no conflict of interest that could have influenced the writing of this text.

\section{References}

1. Hiramoto, K., Yamate, Y., \& Sato, E. F. (2021). p53 and clock genes play an important role in memory and learning ability depression due to long-term ultraviolet A eye irradiation. Photochemical and Photobiological Sciences, 20(5), 677-685. https:// doi.org/10.1007/s43630-021-00055-5

2. de Assis, L. V. M., Tonolli, P. N., Moraes, M. N., Baptista, M. S., \& Castrucci, A. M. L. (2021). How does the skin sense sun light? An integrative view of light sensing molecules. Journal of Photochemistry and Photobiology C Photochemistry Reviews, 47, 100403. https://doi.org/10.1016/j.jphotochemrev.2021.100403

3. Mahmoud, B. H., Ruvolo, E., Hexsel, C. L., Liu, Y., Owen, M. R., Kollias, N., Lim, H. W., \& Hamzavi, I. H. (2010). Impact of longwavelength UVA and visible light on melanocompetent skin. The Journal of Investigative Dermatology, 130(8), 292-297. https:// doi.org/10.1038/jid.2010.95

4. Mahmoud, B. H., Hexsel, C. L., Hamzavi, I. H., \& Lim, H. W. (2008). Effects of visible light on the skin. Photochemistry and Photobiology, 84(2), 450-462. https://doi.org/10.1111/j.17511097.2007.00286.x

5. Hiramoto, K., Yamate, Y., Sugiyama, D., Matsuda, K., Iizuka, Y., \& Yamaguchi, T. (2018). Tranexamic acid inhibits the plasma and non-irradiated skin markers of photoaging induced by long-term UVA eye irradiation in female mice. Biomedicine and Pharmacotherapy, 107, 54-58. https://doi.org/10.1016/j.biopha.2018.07. 146

6. de Assis, L. V. M., Moraes, M. N., \& Castrucci, A. M. L. (2019). The molecular clock in the skin, its functionality, and how it is disrupted in cutaneous melanoma: A new pharmacological target? Cellular and Molecular Life Sciences, 76(19), 3801-3826. https:// doi.org/10.1007/s00018-019-03183-5

7. de Assis, L. V. M., \& Oster, H. (2021). The circadian clock and metabolic homeostasis: Entangled networks. Cellular and Molecular Life Sciences, 78(10), 4563-4587. https://doi.org/10.1007/ s00018-021-03800-2 Epub ahead of print. 